\title{
3D ChemiSTEMTM Tomography of Nano-scale Precipitates in High Entropy Alloys
}

\author{
J.M. Sosa ${ }^{1}$, D.E. Huber ${ }^{1}$, B. Welk ${ }^{1}$, J.K. Jensen ${ }^{1}$, R.E.A. Williams ${ }^{2}$, S. Lambert ${ }^{2}$, H.L. Fraser ${ }^{1}$ \\ ${ }^{1}$ Center for the Accelerated Maturation of Materials, The Ohio State University, Columbus \\ ${ }^{2}$ Center for Electron Microscopy and Analysis, The Ohio State University, Columbus
}

Electron tomography is a three-dimensional technique well suited for characterizing fine-scale microstructural features on the order of 10-100 nm. Typically performed in a transmission electron microscope (TEM), it relies on successive image acquisition at multiple sample tilts. Images may be formed through various techniques including conventional and scanning transmission electron microscopy (CTEM and STEM), energy-filtered TEM (EFTEM), and energy dispersive x-ray spectroscopy (EDS). The variety of available image formation mechanisms has defined electron tomography as a robust fine-scale characterization tool.

Conventional TEM tomography has relied on diffraction contrast as the primary imaging mode. However, this contrast may be insufficient for discriminating different phases in a complex microstructure. In these cases, more informative contrast may be obtained through compositional mapping, where characteristic x-ray emission is recorded over an area of interest. FEI's recently developed ChemiSTEM ${ }^{\mathrm{TM}}$ technology employs a detection configuration with four silicon drift detectors (SDD), providing a very large collection angle $(0.8 \mathrm{str})$, coupled with high incident beam current, resulting in the ability to collect x-ray maps on the order of electron micrographs [1]. Despite such efficiency, few tomographic reconstructions have employed ChemiSTEM ${ }^{\mathrm{TM}}$ mapping as the primary imaging mode. This paper will present novel microstructural characterization of the emerging material system of high-entropy alloys (HEAs) using

ChemiSTEM ${ }^{\mathrm{TM}}$ tomography.

HEAs offer an attractive balance of properties including high strength and corrosion resistance [2, 3]. These materials often involve microstructures that result from phase separation and also spinodal decomposition, such that the resulting microstructures are three-dimensionally interconnected. It is important that the true distribution of these phases be established so that accurate models of the deformation processes may be developed, and hence it is necessary to invoke direct 3D characterization. The size-scale of the microstructural features $(<100 \mathrm{~nm})$ is well suited for electron tomography. Rather than acquire a tilt-series from a thin foil, a needle-shaped specimen was excised using a DualBeam ${ }^{\text {TM}}$ FIB/SEM, which offered several advantages. Firstly, when tilted about its longitudinal axis, the needle's symmetry avoided the projected thickness variations that occur when tilting thin foils. Secondly, the gradation of the needle's thickness allowed the authors to explore the limits of various reconstruction algorithms and evaluate their efficacy as a function of sample thickness.

For this work, a FEI Titan 60-300 ChemiSTEMTM equipped with a quad-detector SDD was used to collect a tomographic tilt series of EDS spectral images from the aforementioned needle of HEA microstructure. Incident beam dwell times were $20 \mu \mathrm{s} / \mathrm{px}$, with a live time of $600 \mathrm{~s}$. The tomographic tilt series was collected about an axis parallel to the needle from $-62^{\circ}$ to $+62^{\circ}$ in $1^{\circ}$ increments. In order to collect adequate signal, beam currents of $\sim 2 \mathrm{nA}$ were used. Beam amperages of this magnitude can threaten a thin specimen's integrity, especially when repeated imaging of the same local area is required, as with tomography. Therefore, one objective of this work was to optimize the sampling frequency (i.e. tilt step size) so as to minimize specimen damage while still obtaining a high-fidelity reconstruction. 
This particular HEA alloy comprised an overall composition of $\mathrm{CoCrNiFeCuAl}_{2.1}$ and a microstructure consisting of three phases rich in $\mathrm{Cr}, \mathrm{Cu}$, and $\mathrm{Ni}$, respectively. As such, ChemiSTEM ${ }^{\mathrm{TM}}$ maps of these elements provided strong phase contrast for tomographic reconstruction. Figure 1a-c displays $-62^{\circ}$ tilt elemental maps of $\mathrm{Cr}$ and $\mathrm{Cu}$ along with a corresponding HAADF micrograph. Following their collection, each series required rigid tilt-to-tilt image registration involving both translation and rotation; this was critical for accurate 3D reconstruction. A weighted back-projection was then performed on each of the aligned series of elemental maps, which resulted in three reconstructed volumes. Each volume required its own segmentation routine to identify the salient microstructural features. A visualization of the three-dimensionally reconstructed $\mathrm{Cr}$-rich and $\mathrm{Cu}$-rich phases is shown in Fig. 1d. Among else, the reconstructions revealed the orthogonal orientations of the $\mathrm{Cu}$-rich platelets as well as their consistent proximity to the Cr-rich precipitates. Neither of these observations was concluded from the alloy's previous two-dimensional characterization. This work has been supported by the DAGSI program of the State of Ohio and the Air Force Research Laboratory.

\section{References:}

[1] P. Schlossmacher, D.O. Klenov, B. Freitag, S. von Harrach, A. Steinbach, Nanoscale Chemical Compositional Analysis with an Innovative S/TEM-EDX System, Microsc. Anal. Nanotechnol. Suppl. (2010) 5.

[2] J.-W. Yeh, S.-K. Chen, S.-J. Lin, J.-Y. Gan, T.-S. Chin, T.-T. Shun, et al., Nanostructured HighEntropy Alloys with Multiple Principal Elements: Novel Alloy Design Concepts and Outcomes, Adv. Eng. Mater. 6 (2004) 299-303.

[3] X. Yang, Y. Zhang, P.K. Liaw, Microstructure and Compressive Properties of NbTiVTaAlx High Entropy Alloys, Procedia Eng. 36 (2012) 292-298.
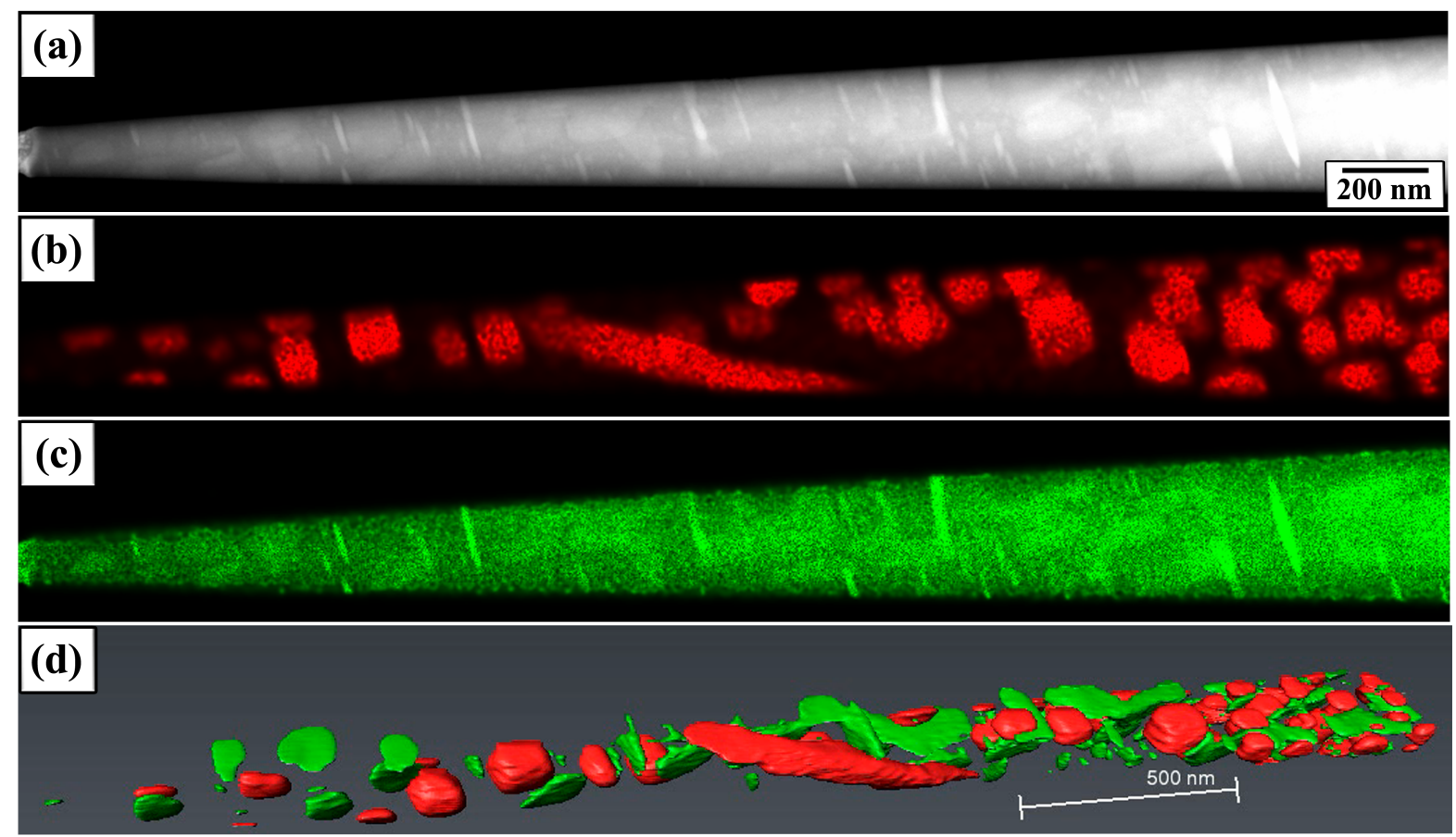

Figure 1. (a) HAADF micrograph with ChemiSTEM ${ }^{\mathrm{TM}}$ elemental maps of (b) $\mathrm{Cr}$ and (c) $\mathrm{Cu}$ at $-62^{\circ}$ tilt as well as (d) a 3D reconstruction of the Cr-rich (red) and $\mathrm{Cu}$-rich (green) phases. 